\title{
CDK9 Inhibitor AZD4573
}

National Cancer Institute

\section{Source}

National Cancer Institute. CDK9 Inhibitor AZD4573. NCI Thesaurus. Code C153377.

A selective, short-acting inhibitor of the serine/threonine cyclin-dependent kinase 9 (CDK9), the catalytic subunit of the RNA polymerase II (RNA Pol II) elong ation factor positive transcription elong ation factor b (PTEF-b; PTEFb), with potential antineoplastic activity. Upon intravenous administration, AZD4573 binds to and blocks the phosphorylation and kinase activity of CDK9, thereby preventing PTEFb-mediated activation of RNA Pol II, leading to the inhibition of gene transcription of various antiapoptotic proteins. This induces cell cycle arrest and apoptosis, and leads to a reduction in tumor cell proliferation. CDK9 regulates elong ation of transcription through phosphorylation of RNA polymerase II at serine 2 (p-Ser2-RNAPII). It is upregulated in various tumor cell types and plays a key role in the regulation of Pol II-mediated transcription of anti-apoptotic proteins. Tumor cells are dependent on anti-apoptotic proteins for their survival. 